\title{
L'efficacité technique des riziculteurs ivoiriens : la vulgarisation en question
}

Technical efficiency of Ivorian rice producers: rural extension services talking about

\section{Ekou Nuama}

\section{OpenEdition}

Journals

\section{Édition électronique}

URL : http://journals.openedition.org/economierurale/2598

DOI : 10.4000/economierurale. 2598

ISSN : 2105-2581

\section{Éditeur}

Société Française d'Économie Rurale (SFER)

\section{Édition imprimée}

Date de publication : 5 avril 2010

Pagination : 36-47

ISSN : 0013-0559

Référence électronique

Ekou Nuama, «L'efficacité technique des riziculteurs ivoiriens : la vulgarisation en question », Économie rurale [En ligne], 316 | mars-avril 2010, mis en ligne le 05 avril 2012, consulté le 01 mai 2019. URL : http://journals.openedition.org/economierurale/2598; DOI : 10.4000/economierurale.2598 


\section{L'efficacité technique des riziculteurs ivoiriens La vulgarisation en question}

Ekou NUAMA • UFR de Sciences Économiques et de Gestion, Université de Cocody, Côte d'Ivoire, et Chercheur au Cires

\section{La problématique}

$\mathbf{T}$ ant par le nombre de personnes qui en vivent que par sa contribution au Produit intérieur brut (PIB), l'agriculture représente la clé de voûte de l'économie ivoirienne. En effet, elle génère en moyenne 30 à $35 \%$ du PIB et constitue la principale source de revenu et d'emploi pour plus de $60 \%$ de la population active (Banque mondiale, 2003). Elle a deux grandes composantes : l'agriculture d'exportation et l'agriculture vivrière. La première constitue le fer de lance de l'économie ivoirienne tant par l'activité industrielle intérieure qu'elle suscite que par l'importance des exportations qu'elle génère. La seconde composante, qui sert à l'alimentation de la population, est principalement dominée par cinq cultures, à savoir : l'igname, le manioc, la banane plantain, le maïs et le riz. À l'exception du riz, la Côte d'Ivoire est pratiquement autosuffisante pour les produits alimentaires à grande consommation (banane plantain, igname, manioc, etc.). Malgré d'importantes potentialités pour la production du riz, la Côte d'Ivoire dépend encore à près de $60 \%$ des importations. Ces importations sont dues à la forte demande de riz en milieu urbain, consécutive à une intense urbanisation et à un changement des habitudes alimentaires des citadins (Nuama et Yoli, 2008). Depuis quelques années, le riz prend une part de plus en plus importante dans l'alimentation des ménages urbains ivoiriens. Il est devenu une denrée de première nécessité dont la consommation croît très rapidement sous le double effet de l'augmentation de la population et de la consommation par tête d'habitant notamment en milieu urbain.

La faiblesse de l'offre de riz pourrait être liée à l'inadéquation des incitations à la production et au manque d'efficacité des riziculteurs. Concernant les incitations à la production de riz, l'État ivoirien est intervenu dans le secteur en apportant son appui aux agriculteurs en matière de formation, d'organisation professionnelle, d'aménagement des périmètres uniquement pour le riz irrigué et pour la mobilisation des financements. La production nationale, qui est évaluée selon les statistiques du ministère de l'Agriculture et des Ressources animales en 2005 à 709931 tonnes, ne parvient pas à couvrir la consommation totale intérieure de riz. Le taux de couverture se maintient autour de 50 à $60 \%$ chaque année avec des importations annuelles qui ont varié de 735759 tonnes en 2003 à 808205 tonnes en 2005 (tableau 1).

Malgré les efforts de l'État, la production domestique de riz est largement en deçà de la consommation locale. Ainsi, il convenait de se poser la question de savoir pourquoi la Côte d'Ivoire continue à importer du riz,

Tableau 1. Évolution de la production et des importations de riz en Côte d'Ivoire

\begin{tabular}{|c|c|c|c|}
\hline Variables $\quad$ Années & 2003 & 2004 & 2005 \\
\hline Production (en tonnes) & 659824 & 681521 & 709931 \\
\hline Superficie (en hectares) & 339936 & 340956 & 341976 \\
\hline Importations (en tonnes) & 735759 & 715379 & 808205 \\
\hline
\end{tabular}

Source : données du ministère de l'Agriculture et des Ressources animales, 2005 
alors qu'elle a tout ce qu'il faut pour en produire suffisamment. Cet article s'intéresse uniquement au deuxième aspect des causes de la faiblesse de l'offre de riz et vise à analyser les obstacles à la production de cette denrée. Comment accroître la production de riz sans engendrer d'autres charges supplémentaires ? Une des réponses à ces interrogations est d'évaluer d'abord le niveau de performance et ensuite d'examiner les déterminants de la performance productive dans la production de riz local en vue d'identifier les sources d'inefficacité. En fait, il s'agit d'examiner les facteurs permettant de réduire leur inefficacité productive. En outre, l'analyse des déterminants de l'efficacité technique est intéressante dans la mesure où elle permet de révéler d'autres sources de croissance de la production de riz sans engendrer de coût supplémentaire pour les exploitants. L'amélioration de l'efficacité productive des riziculteurs permettrait d'accroître éventuellement leur revenu, de réduire d'une part l'insécurité alimentaire en milieu paysan et, d'autre part, les importations massives de riz et d'améliorer la balance commerciale du pays.

Trois sections structurent cette étude. Après avoir présenté un aperçu des notions de frontière de production et d'inefficacité technique, le modèle d'analyse et la collecte des données seront exposés, permettant ainsi l'identification de résultats.

\section{Les notions de frontière de production et d'inefficacité}

Cette section définit la frontière de production et l'inefficacité technique et analyse quelques références bibliographiques sur les déterminants de l'inefficacité technique. La frontière de production indique la quantité maximale de produits obtenue à chaque combinaison d'intrants et l'on ne peut examiner les déterminants de l'efficacité productive que si on l'a préalablement estimée.

La notion d'efficacité comporte dans la littérature économique deux grandes compo- santes : l'efficacité technique et l'efficacité allocative.

\section{La première composante de l'efficacité} se réfère à l'organisation matérielle de la production. Selon Boussemart et Dervaux (1994), à dotation factorielle donnée, l'efficacité technique d'une entreprise se mesure comme l'écart existant entre le niveau de production observé et le niveau de production maximal déterminé par la frontière de production. Un exploitant est techniquement efficace si, pour un niveau de facteurs et de produits utilisés, il est impossible d'augmenter la quantité d'un produit sans augmenter la quantité d'un ou plusieurs facteurs ou de réduire la quantité d'un autre produit. L'exploitant techniquement le plus efficace est celui qui, à niveau de production égal, a utilisé le moins d'intrants ou à niveau d'intrant égal, a obtenu plus de production. L'inefficacité technique est le complément à un du score d'efficacité technique. Au plan géométrique, elle est définie comme la distance séparant tout niveau de production observé du niveau maximal qui serait obtenu si tous les intrants étaient utilisés de manière efficace. On dit d'une entreprise qu'elle est techniquement inefficace lorsqu'elle ne se situe pas sur sa frontière de production ; c'est-à-dire qu'avec une quantité déterminée d'intrants, elle n'obtient pas le plus haut niveau de production techniquement réalisable.

La seconde composante de l'efficacité se définit par rapport au système de prix auquel fait face l'exploitant et suivant un comportement d'optimisation économique (minimisation du coût, maximisation du profit). L'exploitant, qui est à la fois techniquement et allocativement efficace, est dit économiquement efficace. Il a un comportement de maximisation de profit. En situation de concurrence, le profit maximal est obtenu quand le revenu marginal de chaque facteur égale son prix et quand le coût marginal de chaque produit égale son prix, de plus, une hypothèse de convexité est nécessaire à l'ensemble de production. 


\section{Méthodes d'estimation des frontières de production}

L'estimation de la frontière de production constitue une étape centrale dans toute analyse de l'efficacité technique et différentes méthodologies ont été développées (Fried et al., 1993). La littérature mentionne deux principales approches : l'approche non paramétrique et l'approche paramétrique. La spécification ou non d'une forme fonctionnelle à la frontière de production est l'élément distinctif de ces deux approches.

Plusieurs méthodes d'estimation de la frontière existent pour estimer les paramètres de l'approche paramétrique. Ces méthodes peuvent être classées selon la forme présumée de la frontière de production ou de coût, selon la technique d'estimation utilisée pour l'obtenir et selon la nature et les propriétés supposées de l'écart entre la production observée et la production maximale.

Aigner et Chu (1968) ${ }^{1}$ ont été les initiateurs des frontières de production paramétriques obtenues par la méthode déterministe selon laquelle l'écart entre la frontière de production et la production observée est dû à l'inefficacité de l'exploitant. L'inconvénient de cette méthode est qu'elle ne prend pas en compte les phénomènes aléatoires qui peuvent influencer le niveau d'efficacité.

La nature des écarts entre la production observée et la production maximale différencie les frontières stochastiques des frontières déterministes. Le choix entre la frontière stochastique et la frontière déterministe est purement optionnel. Il dépend de l'analyse que le chercheur fait de la situation. Certains chercheurs supposent que tout écart observé est uniquement dû à l'inefficacité du producteur, et ils qualifient la frontière de nature déterministe. $\mathrm{Si}$, en revanche, les chercheurs estiment que les écarts sont expliqués à la fois par le manque d'efficacité du producteur et par des éléments aléatoires

1. Voir Murillo-Zamorano (2004). qui échappent au contrôle de l'exploitant, on dit que la frontière de production ou de coût est de nature stochastique.

L'effet aléatoire a été introduit par Aigner et al. (1977) et Meeusen et Van den Broek (1977) pour tenir compte des facteurs qui échappent au contrôle de l'exploitant. L'estimation de la frontière de production stochastique peut se faire à l'aide des méthodes économétriques (la méthode des moindres carrés corrigés et la méthode du maximum de vraisemblance).

L'approche non paramétrique a la particularité de n'imposer aucune forme préétablie à la frontière de production ou de coût. Les scores d'efficacité sont obtenus par des techniques d'optimisation. Farrell (1957) fut le premier à proposer la frontière non paramétrique convexe dans le cadre d'une étude visant à envelopper les activités de production observées de manière à ce que l'ensemble des possibilités de production ainsi formé soit convexe. L'évaluation des indices d'efficacité est obtenue par la technique d'optimisation (la programmation linéaire ou quadratique) qui minimise l'écart entre la production observée et la production optimale.

\section{Déterminants de l'efficacité}

Conformément aux travaux de Balcombe et al. (2008) et de Battese et Coelli (1995), les déterminants de l'efficacité technique généralement utilisés en agriculture sont le crédit, l'encadrement agricole, le nombre d'années d'expérience de l'exploitant, son âge, son niveau d'instruction, la taille de l'exploitation et l'entraide, etc.

Les effets du crédit et de l'instruction sur la productivité des agriculteurs ont fait l'objet de controverses, pour certains chercheurs, ils améliorent la performance agricole. En revanche, pour d'autres, ils n'ont aucun effet sur l'efficacité technique des producteurs agricoles. L'instruction évaluée en termes de nombre d'années d'études est une variable qui devrait améliorer l'efficacité des exploitants agricoles dans le sens 
qu'un producteur agricole instruit a facilement la maîtrise des techniques modernes de production (Battese et Coelli, op. cit. ; Coelli et Fleming, 2004). Mais en Côte d'Ivoire, les travaux d'Audibert et al. (1999) et de Gurgand (1993) ont montré le contraire. Pour eux, les ménages les plus instruits réduisent la part de l'agriculture dans leur activité, pour se concentrer sur des emplois plus rémunérateurs ou plus prestigieux. L'accès au crédit permet d'accrô̂tre l'efficacité des agriculteurs par l'amélioration de leur aptitude à prendre des risques, ils peuvent par exemple entreprendre dans le cas des cultures vivrières, des cultures de contre saison qui ont une grande valeur marchande. Le crédit permet l'acquisition d'intrants coûteux mais nécessaires à la production. Si les fonds obtenus par les paysans à travers les structures de prêts informelles ou formelles servent à l'achat d'intrants modernes, le crédit influence positivement l'efficacité des exploitants (Nuama, 2006 ; Hazarika et Alwang, 2003). Cependant, le crédit peut ne pas avoir d'effet sur la performance agricole, s'il est utilisé à d'autres fins (Nyemeck et al., 2004).

Dans un autre registre, Thiam et al. (2001) ont étudié à l'aide d'une méta-analyse les facteurs qui influencent l'efficacité technique dans les pays en développement. L'analyse a porté sur un échantillon de 51 observations à travers 32 études. Le principal résultat est d'une part que la forme fonctionnelle de type Cobb-Douglas et l'utilisation des données de coupe transversale ont tendance à minimiser le niveau d'efficacité technique. Cependant, d'autres éléments tels que l'ajout de nouvelles variables au modèle, la fonction de production, la nature de la frontière stochastique ou déterministe et la taille de l'échantillon n'affectent pas significativement les scores d'efficacité technique.

L'entraide en agriculture consiste à réduire la contrainte en main d'œuvre en période de pointe. Boussard (1987) montre que l'entraide engendre des gains de productivité importants dans la mesure où elle intervient à un moment où la productivité de chaque minute gagnée est importante.

\section{Le modèle d'analyse et la collecte des données}

Le modèle utilisé pour évaluer le niveau d'inefficacité technique des exploitations de riz est la frontière de production stochastique encore appelée frontière de production à erreurs composées et à effets d'inefficacité incorporés proposée par Battese et Coelli (op.cit). Ce modèle a été introduit dans la littérature par Aigner et al. (op.cit) et Meusen et Van den Broeck (op.cit). L'avantage de ce type de frontière de production est qu'il permet d'expliquer les déviations observées entre la production maximale et la production réellement obtenue par l'exploitant ainsi que les effets des facteurs aléatoires qui échappent au contrôle de l'exploitant.

Mathématiquement, si l'on considère un riziculteur qui combine des facteurs de production (superficie, main d'œuvre, capital) pour produire un bien Y (le riz), la frontière de production stochastique est représentée par la formule suivante :

$$
\begin{aligned}
& \operatorname{LnY}_{\mathrm{i}}=\operatorname{Lnf}\left(\mathrm{X}_{\mathrm{i}} ; \beta\right) \exp \left(\mathrm{V}_{\mathrm{i}}-\mathrm{U}_{\mathrm{i}}\right) \\
& \mathrm{U}_{\mathrm{i}}=\delta_{0}+\sum \delta_{\mathrm{i}} \mathrm{Z}_{\mathrm{i}}+\mathrm{W}_{\mathrm{i}},
\end{aligned}
$$

i représente les riziculteurs, il varie de 1 à $\mathrm{N}$, Ln : est le logarithme népérien,

Exp : est la fonction exponentielle ;

$\mathrm{Y}_{\mathrm{i}}$ : est la production du ième riziculteur au cours de la campagne ;

f : est la frontière de production ;

$\mathrm{X}_{\mathrm{i}}$ : est le vecteur des intrants du ième riziculteur ;

$\beta$ : est le vecteur des paramètres inconnus de la frontière ;

$\delta$ : est le vecteur des paramètres inconnus des déterminants de l'efficacité ;

$\mathrm{N}$ : représente la taille de l'échantillon; $\mathrm{V}_{\mathrm{i}}$ : est le terme d'erreur symétrique habituel ; c'est un terme purement résiduel prenant en compte les variations de la production de riz qui ne sont pas sous le 
contrôle du riziculteur (il peut, en effet, y avoir des facteurs externes tels le climat, la chance, etc. qui peuvent expliquer que la production ne se trouve pas exactement sur la frontière efficace, c'est cette composante qui donne une interprétation stochastique à la frontière) ; ce terme peut aussi, comme c'est traditionnellement le cas, tenir compte des erreurs d'observations ou des variables manquantes dans le modèle ;

$\mathrm{U}_{\mathrm{i}}$ : est le terme d'erreur non négatif représentant l'inefficacité technique de l'exploitant $i$, cette composante reflète le fait que chaque unité de production doit se trouver en dessous de la frontière de production.

La frontière de production définie au niveau du modèle (1) est stochastique dans le sens où elle combine les deux termes d'erreur $U_{i}$ et $V_{i}$. Dans la frontière déterministe, le terme $V_{i}$ n'apparaît pas. Ce type de frontière ne prend donc pas en compte le terme d'erreur classique, et tout écart par rapport à la frontière est considéré comme dû à l'inefficacité.

Les facteurs de production utilisés par le riziculteur i comprennent :

a) la superficie cultivée (en hectares) ;

b) le temps total des travaux des hommes, des femmes et des enfants relatifs aux différentes opérations culturales représente le temps total de travail de la main d'œuvre évaluée en hommes jours, le temps de travail des femmes et des enfants a été converti selon les normes de la FAO en hommes jours équivalents ;

c) le capital (en Francs CFA) est représenté par la valeur des amortissements des équipements utilisés par l'exploitant i, les équipements sont amortis de façon linéaire au taux de $10 \%$.

$\mathrm{Z}_{\mathrm{i}}$ représente les caractéristiques socioéconomiques et la variable «pluri-activité » qui expliquent l'inefficacité technique des riziculteurs que sont:
$\mathrm{Z}_{1}$ : le nombre d'années d'expérience de l'exploitant ;

$\mathrm{Z}_{2}$ : l'instruction de l'exploitant (variable muette), 1 si l'exploitant est instruit et 0 s'il est analphabète ;

$\mathrm{Z}_{3}$ : l'appartenance à un groupement d'entraide (variable muette) 1 s'il appartient au groupe et 0 s'il travaille seul. En effet, au niveau de l'entraide, il ne s'agit plus pour les riziculteurs membres de recruter de la main d'œuvre pour l'exécution des travaux agricoles ; car, ceux-ci disposent globalement de la force de travail suffisante pour effectuer les travaux impliqués par leur plan de production; s'ils travaillent ensemble dans la rizière $i$ aujourd'hui, et le lendemain, ce sera le tour de la rizière $\mathrm{j}$, et ainsi de suite ;

$\mathrm{Z}_{4}$ : l'accès aux services de vulgarisation (variable muette), 1 s'il a un contact avec le service de vulgarisation et 0 si non, le conseiller agricole installé dans les villages centres a à sa charge plusieurs producteurs agricoles. Il divise sa zone de compétence en quatre sous secteurs et il consacre une semaine par sous secteur. Son rôle est d'expliquer les nouvelles techniques de production aux exploitants agricoles en général et aux riziculteurs en particulier, il rend visite aux riziculteurs de sa zone soit en les réunissant sur une rizière témoin ou sur les rizières individuelles. La durée moyenne de ces séances de travail n'excède pas une heure dans les rizières individuelles et deux heures dans les rizières témoins ; $\mathrm{Z}_{5}$ : l'accès au crédit (variable muette), 1 s'il a accès au crédit existant et 0 si non ; $\mathrm{Z}_{6}$ : l'accès à la terre (variable muette), 1 s'il la loue et $0 \mathrm{~s}$ 'il est propriétaire terrien,

$\mathrm{Z}_{7}$ : la nationalité (variable muette), 1 s'il est ivoirien et 0 s'il est un étranger, la riziculture était une activité essentiellement pratiquée par les étrangers maliens et burkinabés et les nationaux se sont intéressés à cette culture dans la zone d'étude que durant ces dernières décennies ;

$\mathrm{Z}_{8}$ : la pluri-activité agricole (variable 
muette), 1 si le riziculteur possède une exploitation de cultures de rente et 0 s'il n'en possède pas ;

$\mathrm{W}_{\mathrm{i}}$ est le terme d'erreur habituel.

Les variables « prix d'achat du kilogramme de riz paddy » et « le genre des riziculteurs » ne figurent pas parmi les déterminants de l'inefficacité, car pour la première variable, les informations statistiques collectées sont des données en coupe transversale. Les riziculteurs font face au même prix fixé au début de la campagne agricole par l'État. Il y a alors une absence de variabilité pour cette variable. Quant à la variable genre, aucune rizicultrice n'était chef d'exploitation, elles travaillaient avec leur époux. De plus, le questionnaire s'adressait au chef de l'unité de production.

Les hypothèses du modèle sont :

(i) $\mathrm{U}_{\mathrm{i}}$ non négatif suit une loi normale de moyenne $\mu$ et variance $\sigma_{u}^{2}$;

(ii) $\mathrm{V}_{\mathrm{i}}$ suit une distribution normale de moyenne 0 et de variance $\sigma^{2}$ et est indépendant de $\mathrm{U}_{\mathrm{i}}$;

(iii) $\mathrm{V}_{\mathrm{i}}$ et $\mathrm{U}_{\mathrm{i}}$ sont indépendants entre eux et indépendants des variables explicatives.

Sur la base des hypothèses précédentes, les paramètres du modèle (1), c'est-à-dire $\beta_{i}, \delta_{i}, \sigma^{2}=\sigma_{\mathrm{u}}^{2}+\sigma_{\mathrm{v}}^{2}$ et $\gamma=\sigma_{\mathrm{u}}^{2} /\left(\sigma_{\mathrm{u}}^{2}+\sigma_{\mathrm{v}}^{2}\right)$ sont estimés par la méthode vraisemblance. Les paramètres $\sigma^{2}$ et $\gamma$ décrivent la contribution de l'efficacité technique à la production; $\sigma^{2}$ est la somme de la variance du terme représentant l'inefficacité et celle du terme aléatoire et $\gamma$ mesure la part du terme d'inefficacité dans la variance totale.

Le degré d'efficacité est obtenu en divisant la production effective par la production correspondante sur la frontière et comme nous avons un modèle sous la forme logarithmique, l'efficacité est donnée par $\exp \left(-\mathrm{U}_{\mathrm{i}}\right)$. Une fois les scores individuels d'efficacité technique obtenus, l'indice d'inefficacité technique individuel est le complément à un du score d'efficacité technique.
Les déterminants de l'inefficacité technique doivent être exogènes et indépendants des facteurs de production. Selon Battese et Coelli $(1993)^{2}$, ces variables peuvent apparaître à la fois dans la frontière de production et dans la fonction des déterminants de l'inefficacité technique. Les déterminants de l'inefficacité technique sont principalement les caractéristiques socio-économiques des exploitants et les caractéristiques des exploitations. Cette approche a été critiquée par Bravo-Ureta et al. (1993) ${ }^{3}$ qui argumentent que ces caractéristiques peuvent avoir un lien direct avec les facteurs de production et un impact direct avec l'estimation des scores individuels d'efficacité. Ainsi, elles devraient être incorporées dans les arguments de la frontière de production ou de coût dans l'estimation des scores d'efficacité au lieu d'intervenir dans l'estimation des déterminants. Notre travail s'inspire de l'étude conduite par Battese et Coelli (1995).

Dans l'estimation de la frontière de production, les facteurs de production sont exprimés en logarithme népérien et les variables socio-économiques et la variable représentant la pluriactivité sont en unité. $\mathrm{La}$ frontière de production est estimée par la méthode du maximum de vraisemblance à l'aide du logiciel « Frontier $4.1 »$ de Coelli (1996). La méthode de maximum de vraisemblance consiste à construire la fonction de vraisemblance puis à déterminer les paramètres qui maximisent cette fonction. Ce logiciel fournit en une seule étape par itération les élasticités de la frontière de production, les scores individuels et le score moyen d'efficacité technique et les coefficients des déterminants.

Pour estimer les paramètres de la frontière de production et ceux des déterminants (les vecteurs $\beta$ et $\delta$ ), nous avons choisi la forme fonctionnelle de type Cobb-Douglas. L'intérêt de cette forme fonctionnelle est qu'elle est pratique et de plus les paramètres de

2. Cité par W. Tian et G. Wan (2000).

3. Voir Johan Stessens (2002). 
cette fonction sont les élasticités de la production vis-à-vis des différents facteurs.

Au niveau des déterminants, pour qu'une variable ait un impact sur l'inefficacité technique, il faut qu'elle soit significativement différente de zéro. De plus, elle réduit l'inefficacité technique si elle a un signe négatif. En revanche, si elle est positive, elle accroît l'inefficacité technique de ces riziculteurs.

Les signes escomptés des déterminants inclus dans le modèle devraient être négatifs. En principe, le riziculteur instruit est techniquement plus efficace que l'analphabète, car il a la possibilité de tirer profit des opportunités de l'encadrement. La variable « accès à la terre par la location » doit être négativement corrélée à l'inefficacité technique, car le riziculteur, qui loue la terre qu'il cultive, doit accroître ses qualités managériales pour produire plus afin d'honorer ses engagements financiers. Il en est de même pour la variable « crédit». La variable « pluriactivité » indique que certains riziculteurs de la zone d'étude possèdent une autre exploitation de culture de rente. Les temps de travaux effectués dans chaque type d'exploitation ont été saisis séparément, et nous nous sommes intéressés uniquement aux temps mis dans les rizières. Le fait qu'un riziculteur, qui possède en plus de son exploitation de riz, une exploitation de culture de rente, l'oblige à partager son temps de travail de l'année entre ses deux types d'exploitation. Concrètement un riziculteur, qui a une exploitation de culture de rente et produit autant qu'un autre qui n'en a pas, toutes choses étant par ailleurs, est considéré comme techniquement plus efficace que l'autre. La variable
« entraide » accroît l'efficacité technique, car elle réduit la contrainte de main d'œuvre pendant les activités culturales. Elle permet de faire face aux pointes de travail.

Les données utilisées dans cette étude sont des données primaires collectées dans les exploitations de riz en Côte d'Ivoire principalement dans les régions de l'Est, du Centre-Est et du Sud-Ouest au cours de la campagne agricole 2006 et l'échantillon contient à la fois des riziculteurs ivoiriens et étrangers (tableau 2). Ces données portent à la fois sur les facteurs de production et sur les caractéristiques socio-économiques et la variable «pluriactivité » des ménages producteurs de riz. Les statistiques sur ces variables sont dans les tableaux I, II, III placés en annexe. Les riziculteurs de notre échantillon ont été tirés de façon aléatoire avec un taux de sondage de $10 \%$ de l'ensemble des riziculteurs recensés par les conseillers agricoles. En effet, un total de 1500 riziculteurs figure sur le fichier de recensement des conseillers de la zone d'étude. Un échantillon de 150 exploitants a été tiré de façon aléatoire ; mais sur les 150 riziculteurs, après l'enquête, 7 fiches n'étaient pas exploitables. En définitive, nous avons obtenu des données complètes sur 143 exploitants de riz (tableau 2).

\section{Les déterminants de la production de riz Discussion des résultats}

Cette section présente les paramètres de la frontière de production, le score moyen d'inefficacité technique des riziculteurs ainsi que les déterminants de la production maximale.

Tableau 2. Répartition des producteurs de riz par région et par nationalité

\begin{tabular}{|c|c|c|c|c|}
\hline Nationalité $\quad$ Région & Est & Centre-Est & Sud-Ouest & Tota \\
\hline Ivoiriens & 32 & 23 & 23 & 78 \\
\hline Burkinabés & 17 & 13 & 13 & 43 \\
\hline Maliens & 8 & 7 & 7 & 22 \\
\hline Total & 57 & 43 & 43 & 143 \\
\hline
\end{tabular}

Source : données d'enquête, campagne agricole 2006 
Tableau 3. Distribution des scores d'efficacité technique

\begin{tabular}{c|c|c}
\hline Score d'efficacité technique & Nombre d'exploitants & Fréquences relatives en \% \\
\hline $0,40-0,50$ & 9 & 6,29 \\
$0,50-0,60$ & 18 & 12,58 \\
$0,60-0,70$ & 31 & 21,68 \\
$0,70-0,80$ & 32 & 22,38 \\
$0,80-0,90$ & 31 & 21,68 \\
$0,90-1,00$ & 22 & 15,39 \\
Total & 143 & 100,00 \\
\hline Moyenne & 0,73 & \\
Médiane & 0,74 & \\
Mode & 0,75 & \\
Écart type & 0,15 & \\
\hline
\end{tabular}

Source : I'auteur, estimation sur la base des données d'enquête de la campagne agricole, 2006

L'analyse des paramètres de la frontière de production révèle que les élasticités de la frontière de production sont respectivement 0,$28 ; 0,31$ et 0,25 pour la superficie, le travail et le capital (tableau 3). Le score moyen de l'efficacité technique des riziculteurs de la zone d'étude est de $73 \%$ (tableau 4), ce qui signifie qu'en moyenne les riziculteurs opèrent à $73 \%$ de leur capacité ; leur degré d'inefficacité est de $27 \%$. Ils peuvent encore accroître la production de riz paddy de $27 \%$ sans coût supplémentaire. La réduction de $27 \%$ d'inefficacité technique serait bénéfique aux riziculteurs et à l'État. Cela permettrait aux zones de production d'accroître probablement le revenu des riziculteurs et réduirait ainsi les importations de riz.

Le modèle estimé est satisfaisant au regard des paramètres estimés (ils sont pour la plupart significatifs) et $\gamma$ est également significatif et compris entre 0 et 1 . L'analyse de la distribution des scores individuels d'efficacité technique montre qu'aucun riziculteur ne situe sur la frontière de production (leur score d'efficacité technique est inférieur à $100 \%$ ) ; environ $15,39 \%$ autres sont très proches de la frontière de production et près de la moitié (47 \%) des riziculteurs sont en deçà du score moyen d'efficacité technique (tableau 3). Ces producteurs techniquement inefficaces devraient faire l'objet d'attention particulière de la part des services d'encadrement en vue de relever significativement leur performance productive.

Le logiciel «Frontier 4.1 » de Coelli (op. cit) permet d'obtenir par itération les paramètres de la frontière de production et les déterminants de l'inefficacité technique. Le signe des paramètres associés aux déterminants de l'inefficacité technique indique l'impact de ceux-ci sur la contre-performance productive. Ainsi, une valeur significativement négative suggère une influence positive et vis-versa. L'analyse des déterminants montre que les facteurs non significatifs c'est-à-dire ceux qui n'ont aucun effet sur la productivité des riziculteurs sont la nationalité, la vulgarisation et l'instruction. La nationalité n'est pas une variable discriminatoire de l'efficacité technique des riziculteurs contrairement à l'idée couramment admise dans la zone d'étude. Quant à la vulgarisation, en principe, elle devrait réduire le niveau d'inefficacité productive mais dans le cas d'espèce, elle a un effet nul sur celui-ci. L'objectif de la vulgarisation est d'améliorer la productivité des cultures vivrières dont la riziculture. En effet, ceci peut être expliqué par le fait que les conseillers agricoles ont à leur charge l'encadrement de toutes les cultures vivrières existantes dans leur sphère de compétence ; leur incompétence s'explique par le fait qu'ils ont trop d'activité.

En ce qui concerne l'effet de l'instruction, elle n'a aucun impact sur la produc- 
tivité car les riziculteurs de l'échantillon sont en majorité analphabètes et ceux qui sont instruits ne représentent que $20 \%$ du total de l'échantillon (tableau II en annexe). Compte tenu de leur nombre réduit, leur effet sur la productivité a été noyé par celui des analphabètes. Si l'échantillon contenait autant de riziculteurs analphabètes qu'instruits, l'effet de l'instruction serait peut-être significatif. De plus, compte tenu de la pénibilité de la production de riz, très peu de personnes instruites s'adonnent à cette activité ; ce qui confirme les travaux de Audibert et al. et de Gurgand réalisés en Côte d'Ivoire. En effet, les résultats de ces études précédemment conduites en Côte d'Ivoire montrent que la scolarisation n'est pas un moyen efficace pour améliorer la production et la productivité agricoles des ménages ruraux. Les ménages ruraux instruits se détournent de l'activité agricole, ils y consacrent moins de travail et produisent moins.
Les variables qui réduisent l'inefficacité technique sont l'appartenance à un groupement d'entraide, le nombre d'années d'expérience, l'accès au crédit, l'accès à la terre par location et la pluriactivité (tableau 4). La variable «nombre d'années d'expérience » améliore l'efficacité technique des riziculteurs, ainsi que la variable «pluriactivité ». Le nombre d'années d'expérience accroît l'aptitude du riziculteur. Plus le riziculteur est expérimenté, plus faible est son niveau d'inefficacité. Quant à la variable «pluriactivité », elle indique que les exploitations de culture de rente et les rizières ne sont pas concurrentes en termes d'utilisation de la main d'œuvre ; mais au contraire les riziculteurs, qui possèdent une exploitation de culture de rente (café ou cacao), utilisent le revenu tiré de ces exploitations de rente pour l'affecter aux activités de la riziculture en ayant recours dans le cas opportun une main d'œuvre salariée. L'accès à la terre

Tableau 4. Les paramètres de la frontière de production et les déterminants

\begin{tabular}{l|c|c|c}
\hline \multicolumn{1}{c|}{ Variables } & Coefficients & Ecart-type & T statistique (Student) $^{4}$ \\
\hline $\begin{array}{l}\text { Frontière de production } \\
\text { Constante }\end{array}$ & 10,01 & 0,7688 & $13,02^{* * *}$ \\
Ln (superficie) & 0,28 & 0,1089 & $2,57^{* * *}$ \\
Ln (travail) & 0,31 & 0,1512 & $2,05^{* * *}$ \\
$\quad$ Ln (capital) & 0,25 & 0,0925 & $2,70^{* * *}$ \\
Paramètres de la variance & & & \\
$\quad \sigma^{2}=\sigma_{u}^{2}+\sigma_{v}^{2}$ & 0,51 & 0,2512 & $2,03^{* * *}$ \\
$\quad \gamma=\frac{\sigma_{u}^{2}}{\sigma_{u}^{2}+\sigma^{2}}$ & 0,95 & 0,5398 & $1,76^{* *}$ \\
Ln (vraisemblance) & $-90,6$ & & \\
Déterminants de l'inefficacité & & & \\
Constante & 0,42 & 0,1935 & $2,17^{* * *}$ \\
Nombre d'années d'expérience & $-0,66$ & 0,1765 & $-3,74^{* * *}$ \\
Instruction & 0,002 & 0,0015 & 1,33 \\
Appartenance à un groupe d'entraide & $-0,10$ & 0,0606 & $-1,65^{*}$ \\
Accès à la vulgarisation & 0,012 & 0,0098 & 1,22 \\
Accès à la terre & $-0,034$ & 0,02 & $-1,70^{*}$ \\
Accès au crédit & $-0,080$ & 0,0488 & $-1,64^{*}$ \\
Nationalité & 0,05 & 0,0323 & 1,55 \\
Culture de rente & $-0,11$ & 0,0595 & $-1,85^{*}$ \\
\hline
\end{tabular}

Source : I'auteur, estimation sur la base des données d'enquête de la campagne agricole, 2006

4. *** significatif à $1 \%$, ** significatif à $5 \%$, * significatif à $10 \%$ 
par location réduit l'inefficacité technique des riziculteurs, car cette forme d'accès à la terre entraîne pour le riziculteur soit des charges à payer au propriétaire terrien, soit le partage de la récolte. Ainsi, le riziculteur a assez d'incitations à être techniquement performant pour honorer ses engagements. Il en est de même pour les riziculteurs qui bénéficient de crédit formel ou informel. L'obtention de crédit réduit certaines contraintes de la production, principalement l'acquisition d'intrants et le recours à une main d'œuvre salariée pour certaines activités culturales. Le riziculteur, qui a bénéficié d'un crédit, quelle que soit sa nature, a intérêt à s'appliquer beaucoup plus pour tirer profit de l'activité de production de riz.

\section{Conclusion}

Cet article avait pour objectif d'identifier les sources d'accroissement de la production de riz en Côte d'Ivoire à partir d'une frontière de production stochastique. Au terme des investigations, il ressort que les principaux déterminants de l'efficacité productive des riziculteurs de notre échantillon sont l'appartenance à un groupement d'entraide, l'accès au crédit, l'accès à la terre par location, la possession d'une exploitation de culture de rente. L'étude a également montré que la vulgarisation telle que pratiquée actuellement par le service d'encadrement du monde rural n'est pas un déterminant efficace de la réduction de l'inefficacité technique des riziculteurs de notre échantillon.
La lecture de ces résultats permet d'émettre des recommandations à deux niveaux : au niveau des services de vulgarisation et au niveau des institutions de crédit et des organisations non gouvernementales.

\section{Les services de vulgarisation}

Bien que leur influence ne soit pas significative, ils doivent chercher à améliorer leur impact ; ainsi, ils devraient revoir leur mode d'encadrement, chercher à intensifier l'encadrement par petits groupes de paysans et pour ce faire ils devraient recruter beaucoup plus de conseillers agricoles pour pouvoir encadrer efficacement les paysans producteurs de cultures vivrières en général et les riziculteurs en particulier. Ils devraient également aider aux regroupements des producteurs en vue de mettre en place des petites coopératives à partir des groupements d'entraide qui existent déjà dans la zone d'étude.

Les institutions de crédit et les organisations non gouvernementales

Elles devraient développer leur action en faveur du monde rural. Ainsi, l'action des Organisations non gouvernementales et des institutions financières décentralisées devrait être encouragée par les pouvoirs publics et elles doivent agir en accord avec le service de vulgarisation, c'est-à-dire fournir un package de services aux riziculteurs composés en partie de moyens de production.

L'auteur tient à remercier vivement les deux referees pour leurs commentaires. 


\section{RÉFÉRENCES BIBLIOGRAPHIQUES}

Aigner D.-J., Chu S.-F. (1968). On estimating the industry production function. American Economic Review, vol. 58, p. 826-839.

Aigner D.-J., Lovell C.-A.-K., Schmidt P. (1977). Formulation and estimation of stochastic frontier production function models. Journal of econometrics, vol. 6 , p. 21-38.

Audibert M., Mathonnat J., Nzeyimana I., Henry M.-C. (1999). Rôle du paludisme dans l'efficience technique des producteurs de coton dans le nord de la Côte d'Ivoire. Revue d'Économie du Développement, volume spécial "Santé et Développement », vol. 4, p. 121-148.

Balcombe K., Fraser I., Latruffe L, Rahman M., Smith I. (2008). Examining sources of technical efficiency in Bangladesh rice farming: An application of a double bootstrap. Applied Economics, vol. 40 (15), p. 1919-1925.

Banque Mondiale (2003). Le Développement durable dans un monde dynamique. Washington D.-C., Rapport mondial sur le Développement.

Battese G.-E., Coelli T.-J. (1993). A stochastic frontier production function incorporating a model for technical inefficiency effects. Australia, Departement of Econometrics, University of New England, Working Papers in Econometrics and Applied Statistics, ${ }^{\circ} 69$, p. 22-30.

Battese G.-E., Coelli T.-J. (1995). A model of technical inefficiency effects in a stochastic frontier production function for panel data. Empirical Economics, vol. 20, p. 325-332.

Boussemart J.-P., Dervaux B. (1994). Diagnostic de l'efficacité productive par la méthode DEA. Application à des élevages porcins. Cahiers d'Économie et Sociologie Rurales, $\mathrm{n}^{\circ} 31$, deuxième trimestre, p. 43-58.

Boussard J.-M. (1987). Économie de l'Agri- culture. Collection Économie agricole et Agro-alimentaire, Économica, février. Coelli T. (1996). A guide to Frontier Version 4.1: A computer program for stochastic frontier production and cost function estimation. Australia, Centre for Efficiency and productivity Analysis, University of New England, midale, NSW, 2351, CEPA Working Paper 96/07.

Coelli T., Fleming E. (2004). Diversification economies and specialisation efficiencies in a mixed food and coffee smallholder farming system in Papua New Guinea. Agricultural Economics, vol. 31, p. 229-239.

Farrell M.-J. (1957) The Measurement of productive Efficiency. Journal of the Royal Statistical Society, Series A, 120, part 3, p. 253-290.

Fried H., Lovell C.-A.-K, Schmidt S.-S. (1993). The measurement of productive efficiency, Techniques and Applications. Oxford, England: Oxford University Press.

Gurgand M. (1993). Les effets de l'éducation sur la production agricole. Application à la Côte d'Ivoire. Revue d'Économie du Développement, vol. 4, p. 37-54.

Hazarika G., Alwang J. (2003). Access to credit, plot size and cost inefficiency among smallholder tobacco cultivators in Malawi. Agricultural Economics, vol. 29, p. 99-109.

Meeusen W., Van den Broeck J. (1977) . Efficiency Estimation from CobbDouglas Production Functions With Composed Error. International Economic Review, vol. 18, p. 435-444.

Murillo-Zamorano L.-R. (2004). Economic Efficiency and frontier Techniques. Blackwell Publishing Ltd, Journal of Economic Survey, vol. 18, n ${ }^{\circ}$ 1, p. 33-77.

Nuama E. (2006). Mesure de l'efficacité technique des agricultrices de cultures 
vivrières en Côte d'Ivoire. Économie rurale, $\mathrm{n}^{\circ} 296$, novembre-décembre, p. 39-52

Nuama E., Yoli Bi Sani Martin (2008). African Imperatives in the New World Trade Order, Case Studies of Agriculture and Food Security. Chapter III: a study of the agricultural sector in Côte d'Ivoire, AERC, p. 22- 52.

Nyemeck B.-J., Tonyè J., Wandji N., Nyambi G., Akoa M. (2004). Factors affecting the technical efficiency among smallholder farmers in a slash and burn agriculture zone of Cameroon. Food Policy, Elsevier, vol. 24, p. 531-545. Stessens J. (2002). Analyse technique et économique des systèmes de production agricole au nord de la Côte d'Ivoire. Thèse de doctorat $n^{\circ} 530$ à la Faculté des Sciences Biologiques Appliquées de la KULeuven, 286 p.

Thiam A., Boris E., Bravo-Ureta, Rivas T.E. (2001).Technical efficiency in developing country agriculture a metaanalysis. Agricultural Economics, vol. 25, p. 235-243.

Tian W., Wan G.-H. (2000). Technical Efficiency and Its Determinants in China's Grain Production. Kluwer Academic Publishers Boston, Manufactured in The Netherlands, Journal of Productivity Analysis, vol. 13, p. 159-179.

\section{ANNEXE \\ Les statistiques des données utilisées dans le modèle}

Tableau I. Statistiques sur les facteurs de production de la riziculture en Côte d'Ivoire

\begin{tabular}{l|c|c|c|c}
\hline Les variables quantitatives & Moyenne & Minimum & Maximum & Écart type \\
\hline La production (en kilogrammes) & 800 & 500 & 1200 & 40 \\
La superficie (en hectares) & 0,75 & 0,50 & 1,5 & 0,05 \\
Le travail (en hommes/jours) & 2154 & 560 & 5460 & 1300 \\
Le capital (en francs CFA) & 6000 & 3500 & 8305 & 2000 \\
\hline
\end{tabular}

Source : I'auteur, données d'enquête, 2006

Tableau II. Statistiques sur les variables socio-économiques des riziculteurs en Côte d'Ivoire

\begin{tabular}{l|c}
\hline Les variables & Proportion (\%) \\
\hline L'accès au crédit & 76 \\
Riziculteurs instruits & 20 \\
Riziculteurs ivoiriens & 55 \\
L'accès à la vulgarisation & 58 \\
La location de terre & 53 \\
Une exploitation de culture de rente & 46 \\
L'appartenance à un groupement d'entraide & 54 \\
\hline
\end{tabular}

Source : l'auteur, données d'enquête, 2006

Tableau III. Statistiques sur les facteurs de production de la riziculture en Côte d'Ivoire

\begin{tabular}{l|c|c|c|c}
\hline Les variables quantitatives & Moyenne & Minimum & Maximum & Écart type \\
\hline Le nombre d'années d'expérience & 13 & 2 & 25 & 5 \\
\hline
\end{tabular}

Source : l'auteur, données d'enquête, 2006 